\section{La môle hydatiforme complète}

\author{
Jean-Jacques Candelier
}

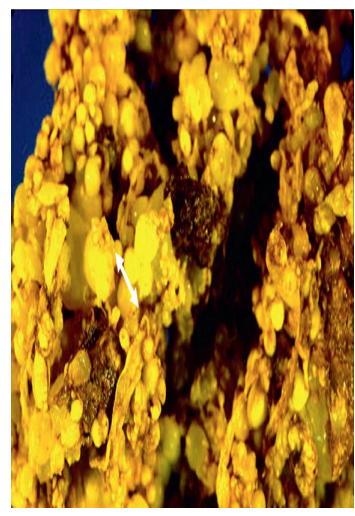

Inserm, unité 1197, Interactions cellules souches-niches physiologie, tumeurs et réparation tissulaire, hôpital Paul Brousse, bâtiment Lavoisier, 14 , avenue Paul Vaillant Couturier, 94800 Villejuif, France ;

et Université Paris-Saclay. jean-jacques.candelier@u-psud.fr

jamais connu le développement de recherche comme d'autres organes qui sont étudiés par les différentes spécialités médicales. II apparaît lorsque, après de multiples divisions cellulaires pendant son trajet dans la trompe utérine, le zygote vient s'implanter (au $6^{\mathrm{e}}$ jour) dans la muqueuse utérine (on parle de blastocyste ${ }^{1}$ ). La couche cellulaire externe de ce blastocyste constitue le trophoblaste. Ce dernier donnera naissance au placenta. Lors de l'invasion de la muqueuse utérine, le trophoblaste se différencie en deux couches : l'une interne, formée du cytotrophoblaste (CTB), et l'autre externe, le syncytiotrophoblaste (STB). Le trophoblaste va progressivement former des villosités dont certaines viendront s'ancrer dans la muqueuse utérine tandis que les autres resteront libres. L'ensemble finira par dessiner un disque plat ressemblant à une sorte de gâteau d'où son nom de placenta (galette en latin). Ces villosités possèdent un axe de tissu lâche, le mésenchyme, richement vascularisé, qui est bordé par une assise de cellules constituée du cytotrophoblaste, dont la prolifération et la différenciation sont à l'origine de la couche externe formée du syncytiotrophoblaste au sein duquel les membranes cellulaires fusionnent (d'où son nom de syncytium) (Figure 1). Ces villosités recouvertes de microvillosités représentent, une fois dépliées, une surface d'environ 14 à $15 \mathrm{~m}^{2}$ constituant une zone d'échanges exceptionnelle. Les villosités libres baignent dans le sang maternel (espace intervilleux) empêchant tout contact entre le sang fœtal et celui de la mère. Elles jouent le rôle de poumon et de rein. Elles permettent aussi la nutrition du fœtus, on parle ainsi de trophoblaste (du grec trophê : nourriture et blastos : germe, feuillet). La prolifération de ce tissu trophoblastique et la colonisation de l'endomètre sont contrôlées à la fois par l'utérus au niveau de la décidue (côté maternel), par des facteurs inhibiteurs comme les TIMP (inhibiteurs tissulaires des

${ }^{1}$ L'embryon au stade blastocyste se compose d'une couche cellulaire externe, et d'une «masse interne », ou bouton embryonnaire, qui donnera naissance aux tissus embryonnaires. 


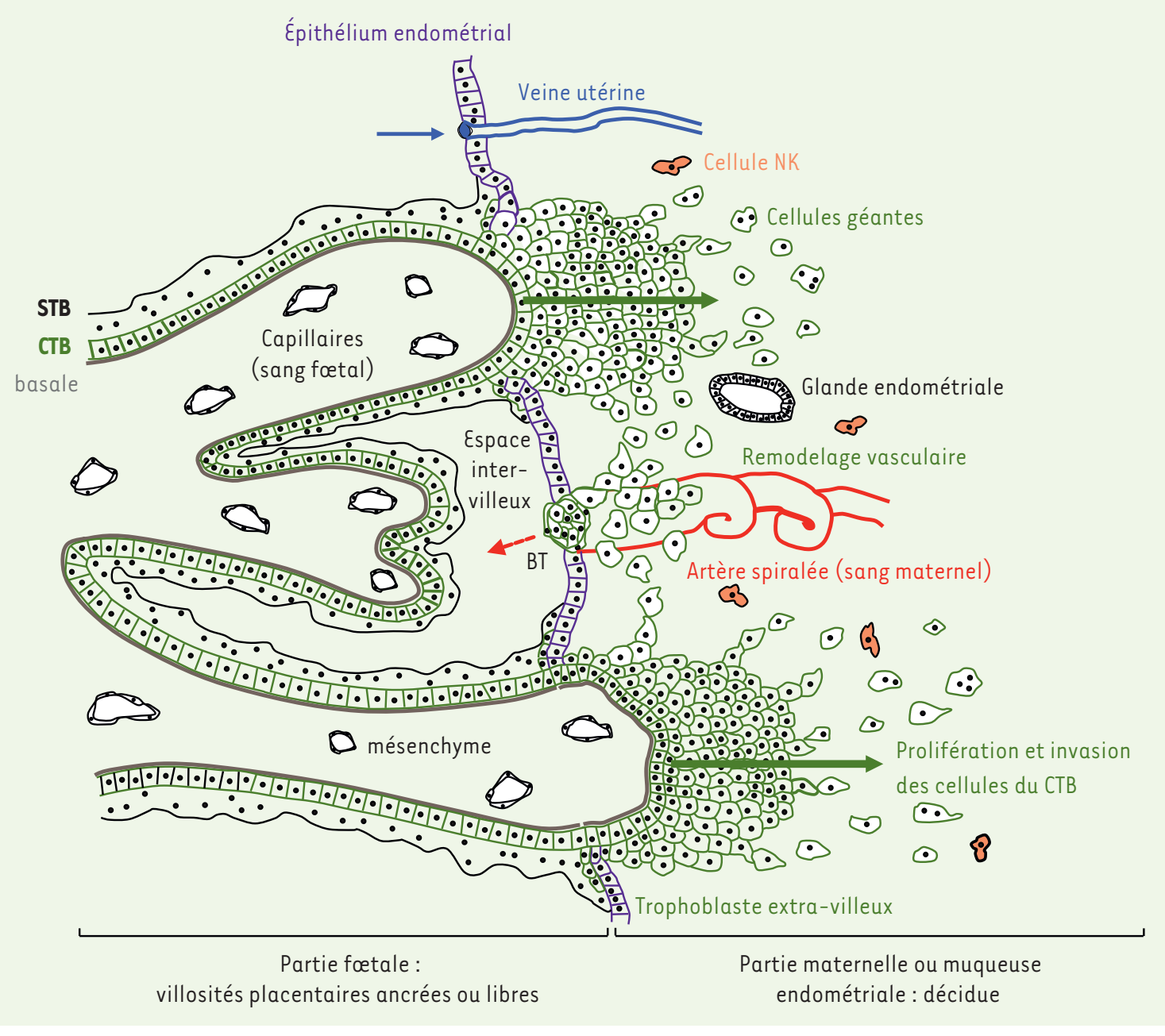

Figure 1. Interface materno-fœtale au niveau du placenta humain au début de son développement. Les cellules du CTB des villosités ancrées se multiplient et envahissent la muqueuse endométriale $(\rightarrow)$. L'invasion des cellules du CTB au niveau des artères spiralées, participe au remodelage progressif de la paroi musculaire de ces dernières, ce qui provoque l'élargissement de ces vaisseaux, on parle alors d'artère utéro-placentaire. C'est au niveau de la lumière de ces mêmes artères que les cellules du CTB formeront les bouchons trophoblastiques (BT). La chambre intervilleuse ne contient donc pas de sang avant 10 à 12 semaines de grossesse. CTB : cytotrophoblaste; STB : syncytiotrophoblaste.

métalloprotéases matricielles) et des facteurs de croissance sécrétés, en particulier par les glandes utérines, mais aussi par le placenta luimême. Les cellules du trophoblaste extravilleux sécrètent (1) des métalloprotéases qui facilitent leur invasion, et (2) la protéine $W_{n t^{2}}$ qui agit, via sa voie canonique, sur certains facteurs de transcription et activerait un ensemble de gènes qui contrôlerait la multiplication, les propriétés invasives et la différenciation des cellules trophoblastiques [2].

Ce dialogue entre les cellules de l'utérus et les cellules trophoblastiques permet aussi aux cellules immunitaires de la mère (macrophages et cellules natural killer, NK) d'être renseignées sur la nature des antigènes tissulaires provenant du père (en particulier les HLA-C)

${ }^{2}$ Wnt est la combinaison de 2 dénominations Wng (Wingless) chez la drosophile, et Int chez la souris. Les Wnt constituent une famille de glycoprotéines qui jouent un rôle dans les développements embryonnaires larvaires et fœetaux, mais aussi dans l'entretien, le renouvellement des tissus adultes. et d'assurer le succès de cette « semi-allogreffe» [3-5]. L'envahissement par les cellules trophoblastiques des artères utérines (artères spiralées) est responsable de leur élargissement dû à une modification progressive de leur paroi musculaire. Il est également à l'origine de la formation des «bouchons » trophoblastiques qui limiteront la circulation sanguine utéro-placentaire pendant le premier trimestre de la grossesse. Cette hypoxie permettra aux tissus embryonnaires et fœtaux de se développer correctement car ils sont sensibles à cet âge au dioxygène et ne possèdent pas d'enzymes antioxydantes. Ce n'est que vers 10 à 12 semaines de grossesse, avec la désagrégation progressive des bouchons trophoblastiques, que la circulation sanguine intervilleuse va réellement se développer et permettre, 


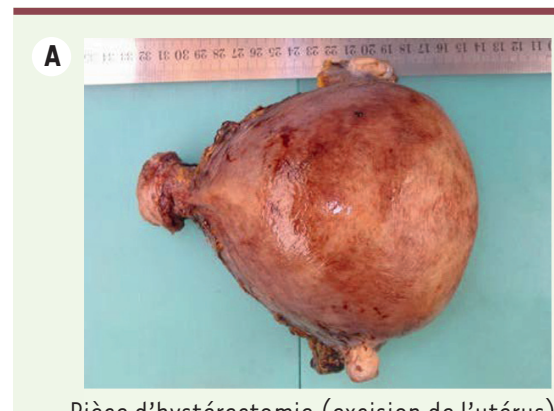

Pièce d'hystérectomie (excision de l'utérus)

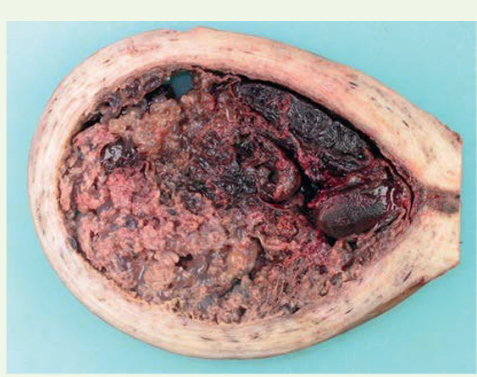

Section longitudinale de l'utérus montrant le développement d'une môle hydatiforme

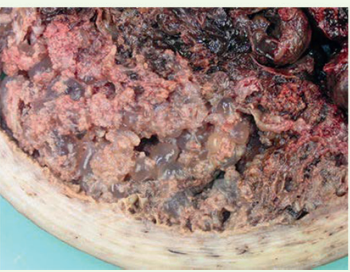

Section montrant les villosités hydropiques

B Macroscopie d'une môle hydatiforme complète et flèche de coupe d'une vésicule hydropique
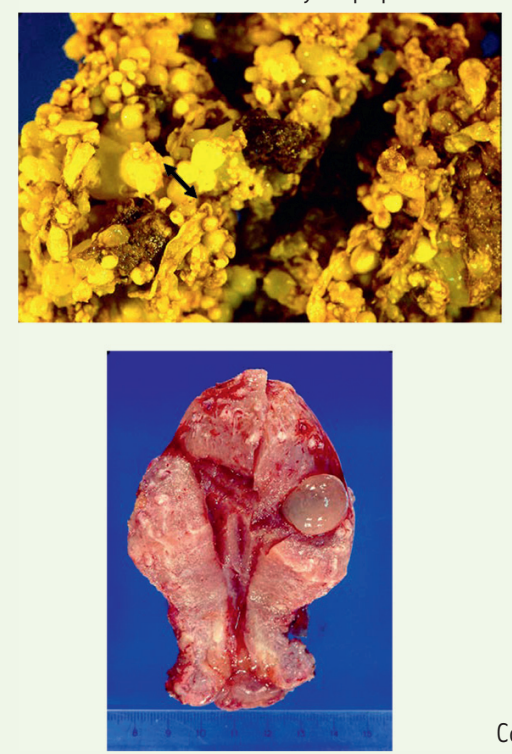

Mole invasive dans le myomètre Pièce d'hystérectomie
Coupe histologique d'une vésicule d'une môle complète selon flèche de coupe précédente Marquage $\beta$-caténine
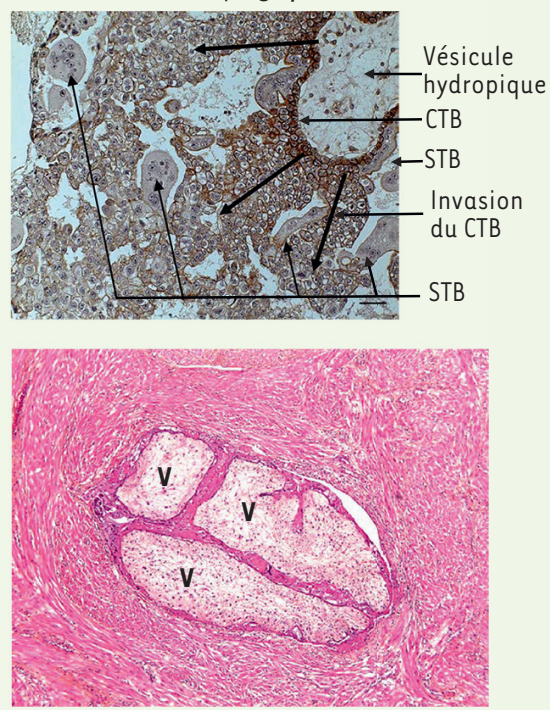

Coupe histologique d'une môle invasive à l'intérieur de la lumière d'un vaisseau

Remarquez les différentes villosités placentaires $(V)$

Figure 2. Macroscopie et histologie des môles hydatiformes. A. Môle hydatiforme in situ. Ces images de prélèvements anatomiques sont dus à la gentillesse des docteurs fœtopathologistes Maryse Bonnière et Bettina Bessières (hôpital Necker, Paris). B. Observation macroscopique et sections histologiques d'une MHC et d'une môle invasive.

lesquelles le trophoblaste prolifère de manière anarchique et forme des vésicules hydropiques ${ }^{3}$. Au cours du premier trimestre de la grossesse, la prolifération de ces vésicules finit par former une masse qui peut ressembler à une « grappe de raisins dilatés » (Figure 2A). L'intérieur de ces villosités dilatées est constitué d'un axe mésenchymateux gorgé de liquide, d'où le terme d'hydropique appliqué à ces tissus (Figure 2B). Au Moyen Âge, on pensait que chaque vésicule représentait une cellule œuf (ou zygote).

Ce placenta môlaire peut comporter des tissus embryonnaires et un certain nombre de villosités normales (on parle alors de môle hydatiforme partielle ou MHP), ou en être dépourvu (on parle dans ce cas de môle hydatiforme complète ou MHC). Ces critères histologiques sont insuffisants pour distinguer entre MHP et MHC quand le développement embryonnaire n'est pas suffisamment avancé, donc au cours $\mathrm{du} 1^{\mathrm{er}}$ trimestre de la grossesse. Quand on analyse ces villosités môlaires, on est surpris par l'absence de vascularisation dans le mésenchyme des villosités les plus hydropiques, alors qu'elle peut être présente dans les villosités

« sans risque », la croissance du fœtus qui, à partir de cette période et jusqu'à la fin de la grossesse, nécessite un apport considérable de nutriments. À l'approche du terme, l'épaisseur du cytotrophoblaste a fortement diminué [6], simplifiant les barrières cellulaires et permettant une nutrition du fœtus encore plus efficace $[7,8]$.

\section{La pathologie môlaire}

\section{Histologie}

Les môles hydatiformes (du grec môles : masse et hydatide : sac hydrique) appartiennent aux pathologies de la gestation dans peu déformées par la pathologie [9]. Cette absence d'alimentation sanguine participerait à l'accumulation de liquide et à la formation du tissu môlaire [10].

\section{Fréquence}

La fréquence des pathologies môlaires peut varier de moins de $1 / 1000$ grossesses dans les pays développés, à plus de $1 / 400$ dans un certain nombre de pays en voie de développement $[11,12]$. Depuis les dernières

\footnotetext{
${ }^{3}$ Structure en grappe de raisin à paroi fine, translucide et kystique.
} 
décennies, ces fréquences ont tendance à baisser [13]. La surveillance médicale et la meilleure nutrition de la mère expliquent la diminution de ces pathologies qui restent, dans $80 \%$ des cas, des tumeurs bénignes dans les pays développés. Les $20 \%$ restants correspondent à des tumeurs malignes (tumeurs trophoblastiques gestationnelles) dont environ la moitié apparaît après une môle hydatiforme [14]. Depuis la seconde moitié $d u x x^{e}$ siècle, la surveillance échographique des grossesses est devenue un examen de routine. La plupart des môles sont désormais détectées dès la première visite échographique, c'est-à-dire avant 12 semaines de gestation. Les écoulements sanguins vaginaux sont, par ailleurs, le symptôme le plus commun de ces pathologies môlaires. À ces observations s'ajoute la détermination, chez la mère, de la concentration sanguine de l'hormone de la gestation (I'hormone gonadotrophique ou HCG). L'HCG étant sécrétée par le syncytiotrophoblaste, dont la croissance est augmentée en cas d'une grossesse môlaire, une concentration plus élevée de HCG est observée. Cette hormone se retrouve dans l'espace intervilleux puis dans la circulation générale de la mère, ce qui facilite son dépistage biochimique. L'ensemble de ces investigations permet de poser le diagnostic de môle et d'envisager, après dilatation du col de l'utérus, l'évacuation du produit de conception par aspiration/curetage. Les tissus môlaires sont alors analysés par les services d'anatomie-pathologique. Les tumeurs trophoblastiques gestationnelles les plus fréquentes correspondent à l'envahissement du myomètre et/ou des vaisseaux utérins, soit sous la forme de villosités (on parle de môle invasive), soit sous la forme de cellules tumorales qui dérivent du cytotrophoblaste (СTB), on parle dans ce cas de choriocarcinome gestationnel. Ces tissus invasifs peuvent être retrouvés dans le myomètre ou à distance, dans les poumons, le foie, le cerveau (Figure 2B) [15]. Le risque de développer une môle invasive ou un choriocarcinome est bien plus important après la survenue d'une môle complète. Ainsi, parmi les patientes porteuses d'une môle complète, 15 à $20 \%$ d'entre elles développeront une môle invasive comparé à moins de $5 \%$ des patientes porteuses d'une môle partielle [16]. Le risque de développer un choriocarcinome est environ 1000 fois plus élevé après la survenue d'une môle complète qu'après tout autre événement gestationnel [17]. Ces pathologies invasives sont agressives et les choriocarcinomes en sont la forme la plus dangereuse. Elles peuvent être fatales dans les pays où le suivi médical n'est pas correctement assuré.

La médicalisation de la naissance en France s'est développée progressivement depuis 1952, période où une femme sur deux était suivie médicalement, jusqu'aux années 1968/1970. Depuis cette date, environ $100 \%$ des femmes ont un suivi [18], permettant un diagnostic précoce et un traitement rapide. Notons que, dans de rares cas, la patiente peut expulser spontanément la môle hydatiforme complète [9].

Mais pourquoi ce placenta se transforme-t-il en une môle complète? Analysons le génome de telles môles.

\section{Caryotype môlaire}

L'analyse cytogénétique des cellules trophoblastiques des môles hydatiformes complètes montre qu'elles contiennent 23 paires de chromosomes $(2 n)$, ce qui correspond au nombre normal de chromosomes d'un être humain. Les techniques de polymorphisme de bandes [19] et de marquage spécifique des chromosomes à l'aide de sondes ciblant certaines séquences d'ADN (technique d'hybridation in situ fluorescente [FISH], développée dans les années 1985/1990) ont mis en évidence une observation inattendue : les $2 \mathrm{n}$ chromosomes observés dans les cellules trophoblastiques correspondent en fait à la duplication du génome haploïde (n) du spermatozoïde. Les chromosomes maternels ont donc disparu et la duplication du génome paternel a rétabli le nombre normal de chromosomes $(n \rightarrow 2 n=$ $46 \mathrm{XX}$ ) (voir plus loin et Figure 3). On parle de génome diploïde par diandrie. Les cellules de ce zygote anormal se multiplient rapidement mais ne donnent naissance qu'à un trophoblaste «bulleux » riche en liquide et dépourvu d'embryon. Cette production de tissus placentaires par un zygote d'origine génétique paternelle (androgenèse) correspond à des observations montrant que l'information génétique paternelle était plus particulièrement responsable de la production des annexes (placenta) et l'information maternelle de la production des tissus embryonnaires [20].

Dans la majorité des cas ( $80 \%$ ), ces môles complètes sont d'origine androgénétique monospermique (fécondation par un seul spermatozoïde) et le caryotype est alors toujours XX (les YY ne sont pas viables) et, dans 10 à $20 \%$ des cas, elles sont androgénétiques dispermiques, le caryotype pouvant être $46 \mathrm{XY}$ ou XX. Dans de rares exemples, ces môles peuvent aussi être diploïdes biparentales (voir ci-dessous), ou triploïdes diandriques dispermiques. En fait, de nombreuses combinaisons chromosomiques ont pu être observées. La présence de deux populations cellulaires de caryotype différent a également été rapportée, on parle alors de môle complète mosaïque [13]. Retenons que dans la majorité des cas, ces môles complètes sont d'origine androgénétique. Ce sont les seules maladies connues dans l'espèce humaine qui se développent par androgenèse $e^{4}$.

\section{Les hypothèses explicatives}

\section{Hypothèses historiques}

Depuis les années 1980, plusieurs modèles théoriques ont été proposés afin d'expliquer la survenue des môles complètes. Par exemple, l'ovocyte est anucléé avant ou

\footnotetext{
${ }^{4}$ Ce texte est consacré aux môles complètes. Les môles partielles représentent un sujet à part entière et sont donc seulement évoquées.
} 


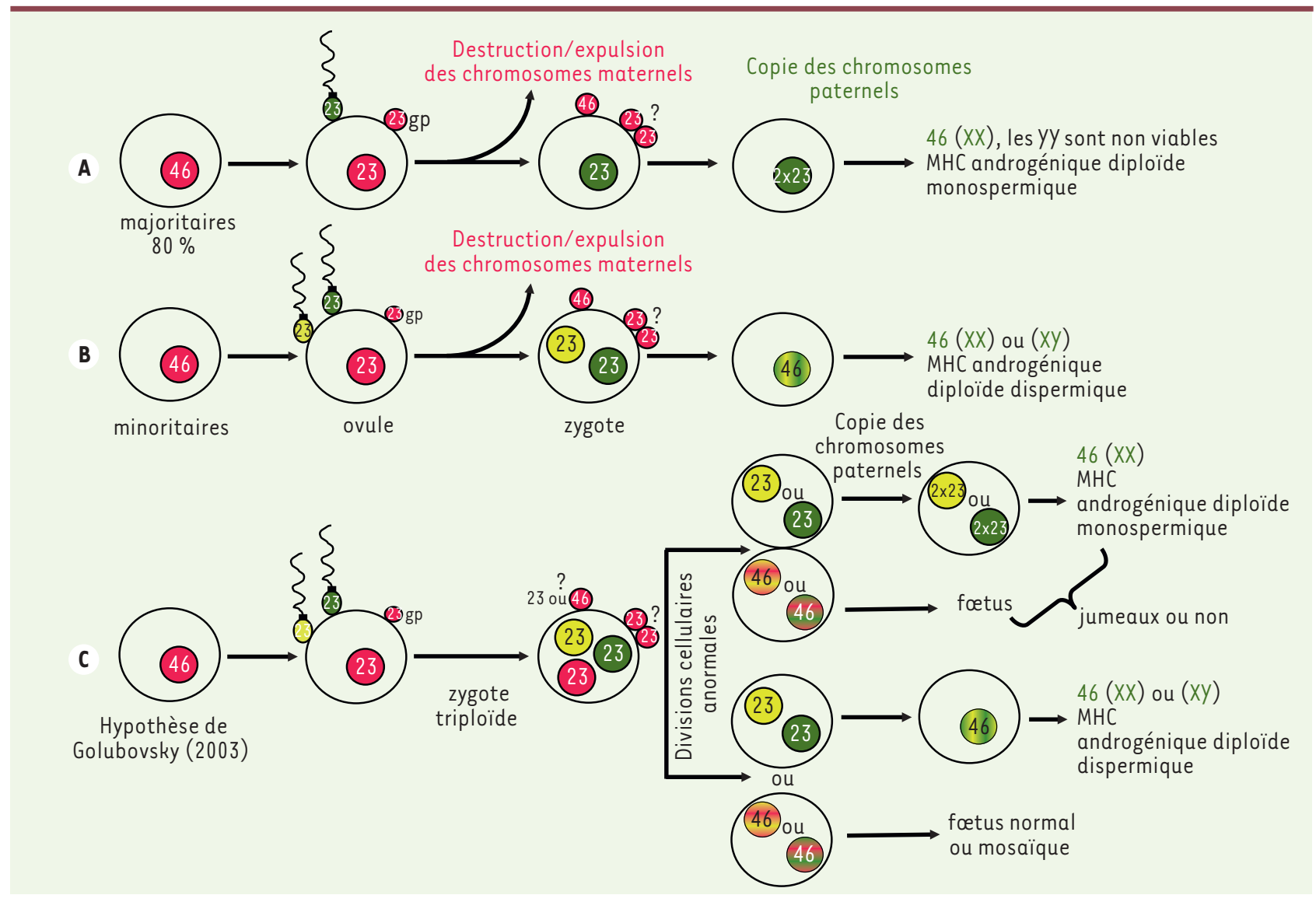

Figure 3. Mécanismes envisagés pour l'origine des chromosomes des môles hydatiformes complètes. A. Après destruction/rejection du noyau de l'ovocyte et endoréplication (copie) des chromosomes paternels on obtient un zygote à 46 chromosomes paternels. Ce zygote est à l'origine d'une MHC monospermique. Le premier globule polaire (gp) peut ou non se diviser et le noyau ovocytaire expulsé contient 23 chromosomes dupliqués (46). B. Même situation qu'en (A) mais deux spermatozoïdes assurent la fécondation. On obtient une MHC dispermique. C. Même situation qu'en (B) mais le pronucléus maternel persiste et c'est la division cellulaire d'un zygote triploïde qui donne naissance soit à des jumeaux monozygotes, soit à une MHC dispermique et un embryon normal. Dans le premier cas, l'un des jumeaux est une MHC par endoréplication des chromosomes de l'un ou l'autre des spermatozoïdes fécondants et l'autre jumeau est un embryon sain par fusion de l'un des pronucléus mâles avec le pronucléus maternel. Dans le second cas, la MHC est obtenue par fusion des deux pronucléus mâles provenant des deux spermatozoïdes fécondants et l'embryon sain par fusion de l'un des pronucléus mâles avec le pronucléus maternel. MHC : môle hydatiforme complète.

après la pénétration du spermatozoïde qui est suivie d'une duplication des seuls chromosomes paternels (Figure $3 A$ ), sauf si deux spermatozoïdes sont fécondants (Figure $3 B$ ). Les ovocytes pourraient aussi présenter une immaturité responsable d'un retard de la division du noyau cellulaire (pronucleus féminin) par rapport au pronucléus mâle. Ainsi, au cours des premières multiplications cellulaires du zygote, cet asynchronisme favoriserait la destruction/rejet des chromosomes maternels. On peut observer, dans quelques rares cas de môles complètes, la persistance d'un seul chromosome maternel parmi les chromosomes paternels dupliqués, argument supplémentaire en faveur de cette hypothèse [21]. Dans d'autres cas, cependant beaucoup plus rares ( $1 \%$ des $M H C$ ), la fécondation a lieu entre un spermatozoïde sain et un ovocyte haploïde, mais le noyau ovocytaire est porteur d'une copie mutée du gène NLRP7 (NLR family, pyrin domain containing 7, également appelé NALP7 ou NOD12) dont 47 mutations différentes ont été décrites, ou du gène KHDC3L ( $K H$ domain containing 3 -like) qui est un gène mineur, sans doute impliqués dans l'intégrité et l'organisation de la région corticale de l'ovocyte et dans la spécification des cellules de l'embryon préimplantatoire vers la formation du trophoblaste ou de l'embryon [22]. II en résulte ainsi des môles hydatiformes complètes diploïdes biparentales, qui sont récurrentes chez une même femme porteuse de ces mutations germinales, ce qui n'est pas le cas des môles androgénétiques les plus communes, qui sont sporadiques et en général non récurrentes ${ }^{5}$.

\footnotetext{
${ }^{5}$ Les môles NLRP7 ou KHDC3L sont les seules actuellement qui sont récurrentes. Les
} autres môles n'ont pas ce statut. 


\section{Hypothèses récentes}

Les hypothèses expliquant la survenue des môles hydatiformes complètes ont été remises en cause en 2003 par Golubovsky [23] (Figure 3C). Dans sa publication, il fait remarquer: (1) qu'il faudrait un « réservoir » régulier d'ovocytes anucléés pour assurer la fréquence des môles hydatiformes complètes; (2) que les ovocytes vides peuvent être obtenus in vitro (mais, dans ce cas, sont-ils viables in vivo et/ou peuvent-ils être fécondés?) (il faut la présence d'ovocytes vides sans les chromosomes maternels pour obtenir une môle complète); (3) que dans les grossesse gémellaires dont un zygote est une môle hydatiforme complète et l'autre est normal, ils sont le plus souvent monozygotes et prédominent alors les môles hydatiformes complètes diploïdes androgénétiques 46XX [24]. Rappelons que dans la première hypothèse évoquée plus haut, on parle d'une disparition rapide des chromosomes maternels dans le zygote et pas dans les ovocytes, ce qui élimine le problème de la réserve d'ovocytes vides. Pour expliquer l'apparition d'une môle complète, Golubovsky propose un ovocyte nucléé, fécondé par deux spermatozoïdes. Pour les jumeaux, lors des premières divisions, le zygote triploïde serait à l'origine d'un clone cellulaire à $2 n$ chromosomes biparentaux et d'un autre à ln chromosomes paternels. Le clone à $2 n$ formerait un fœtus normal, et le clone à ln, après endoréplication des chromosomes, donnerait naissance à une môle hydatiforme complète. Du point de vue épidémiologique, la fréquence de ces triploïdies, qui représentent $1 \%$ de toutes les conceptions, pourrait inclure tous les types de môles hydatiformes. Ce nouveau cadre explicatif laisse penser que l'ovocyte présenterait une réaction corticale anormale, favorisant l'entrée de deux spermatozoïdes (normalement, la libération du contenu enzymatique des granules corticaux interdit l'accès à l'ovocyte à plus d'un spermatozoïde). Ces différentes théories explicatives supposent une altération de la différenciation de l'ovocyte. D'autres observations montrent que l'environnement nutritionnel de la femme pendant sa grossesse peut aussi intervenir.

\section{Épigénétique}

Les modifications interviennent après la fécondation et le début du développement. Elles sont liées à l'environnement dans lequel vit la mère de la patiente atteinte de môle (effet transgénérationnel). Une étude récente de Coullin et ses collaborateurs [25], réalisée au Maroc et au Sénégal où le cycle annuel des saisons est bien marqué, avec une période de diète sévère (durant laquelle peu de produits frais sont disponibles), montre une forte corrélation entre le déficit nutritionnel des mères au moment de la conception de leur fille, et la survenue ultérieure d'une môle hydatiforme complète chez ces filles, lorsqu'elles sont à leur tour enceintes. C'est principalement un déficit en vitamines $A$ et/ou B9 (folates) pendant les premières semaines du développement du fœtus féminin, qui pourrait altérer la différenciation de leurs ovocytes. La vitamine $A$ joue un rôle important dans le bon déroulement de la méiose. Un déficit en cette vitamine est en effet responsable d'une altération de la méiose et donc de la formation d'un ovocyte immature [26]. Quant aux folates, ils sont nécessaires à la synthèse des protéines et de l'ADN, d'où des conséquences possibles de leur déficit, d'une part sur la différenciation de l'ovocyte et du zygote, en particulier sur leur zone corticale comme le suggère le groupe de R. Slim [22], et, d'autre part, sur l'instabilité des chromosomes d'origine maternelle [27]. Lors de la fécondation, le pronucléus mâle poursuivant sa division, les chromosomes instables du pronucléus femelle seraient détruits ou rejetés rapidement. La vitamine $A$ et les folates interviennent aussi dans les mécanismes de la méthylation de I'ADN lors de la reprogrammation des empreintes parentales [28] $(\rightarrow)$. $\rightarrow$ Voir la Synthèse de C. Proudhon et D. Bourc'his, $m / s n^{\circ} 5$, mai 2010, page 497
Contrairement aux autres gènes humains, ces gènes soumis à empreinte (qui représentent environ $1 \%$ du génome) ne s'expriment que de façon monoallélique selon l'origine paternelle ou maternelle des chromosomes du zygote. Dans l'espèce humaine, c'est l'existence de ces gènes soumis à empreinte qui empêche la parthénogenèse [29] et permet le bon développement de l'embryon. De tels déficits nutritionnels durant l'ovogenèse sont responsables d'anomalies qualitatives et fonctionnelles de l'ovocyte [30].

D'autres observations réalisées à Hawaï, où le risque de môle hydatiforme complète est faible, montrent que les femmes récemment immigrées présentent un taux élevé de môle hydatiforme complète. Ces femmes sont natives des Philippines où le taux de môle hydatiforme complète est élevé. Au contraire, les femmes d'origine japonaise, dont la migration est beaucoup plus ancienne, ne présentent plus de risque (le Japon à l'époque, était un pays à haut risque) [31]. Autre situation : entre les années 1974 et 2000, l'incidence des môles hydatiformes au Japon diminue fortement : de 2,79/1000 naissances vivantes en 1976 à 1,61/1000 en 1997. Cette régression est entièrement attribuable à la chute de la fréquence des môles hydatiformes complètes, qui passe de 1,71/1000 naissances vivantes en 1985 à 0,49/1000 en 2000 , et non pas à celle des môles hydatiformes partielles. Or, les môles hydatiformes complètes sont androgénétiques et les môles hydatiformes partielles sont triploïdes diandriques. L'explication la plus pertinente est qu'après les privations de la Seconde Guerre mondiale (entre 1937 et 1945), les conditions économiques se sont progressivement améliorées et la gamétogenèse s'est progressivement normalisée chez les femmes [32], expliquant la diminution des môles hydatiformes complètes. Les môles hydatiformes partielles qui sont principalement le résultat des anomalies du processus gamétogenèse/fécondation dans l'espèce humaine, ne sont pas influencées par de telles variations de l'environnement nutritionnel. 
En résumé, ces exemples que nous évoquons suggèrent que l'apparition des môles hydatiformes complètes est sensible à l'environnement nutritionnel dans lequel se développent les ovocytes du fœtus. C'est ainsi la mère de la patiente présentant une môle qui est à l'origine de cette pathologie et non la patiente! On peut donc parler d'effet transgénérationnel [25].

\section{Conclusion}

L'histoire des môles hydatiformes complètes reste en partie une énigme, un peu à l'image des traitements subis par le placenta au cours de l'histoire des sociétés humaines. Ainsi, il pouvait être consommé dans la France rurale du moyen âge (ingestion du «double de l'enfant », pratique interdite dès le XVIe siècle), ou enseveli (pour un retour à la terre de l'ombre du fœtus) et surmonté d'un arbre (liens entre la nature et l'enfant). Cette dernière pratique a perduré jusqu'au début du XXe siècle. Plus généralement, on apportait à l'accouchée un gâteau rituel garni d'une fève, une sorte de galette des rois. Entre placenta et galette, il n'y aurait donc pas qu'un lien étymologique, mais aussi une survivance tardive de la placentophagie [33]. On retrouve cette idée dans la langue populaire anglaise où être enceinte se dit «have a bun in the oven » (avoir un petit pain dans le four).

Ironie de l'histoire... cet organe aujourd'hui fait beaucoup parler de lui. Il est utilisé comme source de produits cosmétiques, pharmaceutiques, mais aussi comme réservoir de cellules souches mésenchymateuses. Ironie de l'évolution... cet organe si éphémère, que l'on oublie si souvent pour ne s'occuper que du bébé, nous laisse pour toujours un souvenir indélébile. $\diamond$

\section{SUMMARY}

\section{Complete hydatidiform mole}

"The battle of the sexes begins in the zygote" W. Reik and J. Walter [1]. Complete hydatidiform mole (CHM) is a pathology of the placenta with androgenetic diploid origin (chromosomes only from paternal origin). Placental villi present an abnormal hyperproliferation and hydropic degeneration associated with the absence of embryo. Three mechanisms can be envisaged at its origin: (1) destruction/expulsion of the female pronucleus at the time of fertilization by one or two spermatozoa, the former being followed by an endoreplication of the male pronucleus (homozygous mole), (2) a triploid zygote (fertilization by two spermatozoa) leading to a haploid and a diploid clones. The diploid clone may produce a normal fetus while the haploid clone, after endoreplication, generates a complete hydatidiform mole, (3) a nutritional defect during the differentiation of the oocytes of the female embryo that will affect the integrity and maturity of her oocytes during her adult life and lead to hydatidiform mole. In countries with a poor medical health care system, moles can be invasive or, in rare cases, lead to gestational choriocarcinomas. $\diamond$

\section{REMERCIEMENTS}

Madame le Dr Rima Slim, professeur associé et chercheur au McGill University/ McGill University Health Centre Research Institute, a bien voulu relire ce manuscrit et je la remercie très sincèrement ici pour ses conseils.

\section{LIENS D’INTÉRÊT}

L'auteur déclare n'avoir aucun lien d'intérêt concernant les données publiées dans cet article.

\section{RéFÉRENCES}

1. Reik W, Walter J. Evolution of imprinting mechanisms: the battle of the sexes begins in the zygote. Nat Genet $2001 ; 27: 255-6$.

2. Knöfler M, Pollheimer J. Human placental trophoblast invasion and differentiation: a particular focus on Wnt signaling. Front Genet $2013 ; 4$ 1-14.

3. Redman CW, Sargent IL. Immunology of pre-eclampsia. Am J Reprod Immunol 2010 ; 63 : 534-43.

4. Chaouat G. Immunité et grossesse. Med Sci (Paris) 1987 ; $3:$ 599-607.

5. Hiby SE, Walker JJ, O'Shaughnessy KM, et al. Combinations of maternal KIR and fetal HLA-C genes influence the risk of preeclampsia and reproductive success. J Exp Med $2004 ; 200$ : 957-65.

6. Candelier JJ, Frappart L, Diatta AL, et al. Differential expression of $\varepsilon$-cadherin, $\beta$-catenin, and Lewis $x$ between invasive hydatidiform moles and post-molar choriocarcinomas. Virchows Arch 2013 ; $462: 653-63$.

7. Évain-Brion D, Malassiné A. Le placenta humain. Cachan : Éditions Médicales Internationales/Lavoisier, 2010 : 198 p.

8. Malassiné A, Tarrade A, Guibourdenche J, et al. Le placenta. Med Sci (Paris) $2000 ; 16: 329-35$

9. Reynolds SR. Hydatidiform mole: a vascular congenital anomaly. Obstet Gynecol 1976; $47: 244-50$.

10. Kim MJ, Kim KR, Ro JY, et al. Diagnostic and pathogenetic significance of increased stromal apoptosis and incomplete vasculogenesis in complete hydatidiform moles in very early pregnancy periods. Am J Surg Pathol 2006 ; $30: 362-9$

11. Parazzini F, Ricci $\varepsilon$, Cipriani S, et al. Temporal trends in the frequency of hydatidiform mole in Lombardy, northern Italy, 1996-2008. Int J Gynecol Cancer $2012 ; 22: 318-22$.

12. Steigrad SJ. Epidemiology of gestational trophoblastic diseases. Best Pract Res Clin Obstet Gynaecol $2003 ; 17: 837-47$.

13. Slim R, Wallace EP. NLRP7 and the genetics of hydatidiform moles: recent advances and new challenges. Front Immunol $2013 ; 4: 1-12$.

14. Kani KK, Lee JH, Dighe M, et al. Gestational trophoblastic disease: multimodality imaging assessment with special emphasis on spectrum of abnormalities and value of imaging in staging and management of disease. Curr Probl Diagn Radiol 2012 ; 41 : 1-10.

15. Berkowitz RS, Goldstein DP. Chorionic tumors. N Engl J Med 1996 ; 335 : 1740-8.

16. Lurain JR. Gestational trophoblastic disease I: epidemiology, pathology, clinical presentation and diagnosis of gestational trophoblastic disease, and management of hydatidiform mole. Am J Obstet Gynecol 2010; 203 : 531-9.

17. Norvez A. De la naissance à l'école. Santé, modes de garde et préscolarité dans la France contemporaine. Paris: PUF, $1990: 464$ p.

18. Boufettal $H$, Coullin P, Mahdaoui $S$, et al. Les môles hydatiformes complètes au Maroc : étude épidémiologique et clinique.J Gynecol Obstet Biol Reprod $2011 ; 40: 419-29$.

19. Kajii T, Ohama K. Androgenetic origin of hydatidiform mole. Nature 1977 ; $18: 633-4$.

20. Barton SC, Surani MA, Norris ML. Role of paternal and maternal genomes in mouse development. Nature 1984 ; 311 : 374-6.

21. Fisher RA, Nucci MR, Thaker HM, et al. Complete hydatidiform mole retaining a chromosome 11 of maternal origin: molecular genetic analysis of a case. Mod Pathol 2004 ; 17 : 1155-60.

22. Akoury $\varepsilon$, Zhang L, Ao A, et al. NLRP7 and KHDC3L, the two maternal-effect proteins responsible for recurrent hydatidiform moles, co-localize to the oocyte cytoskeleton. Hum Reprod 2015 ; 30 : 159-69.

23. Golubovsky M.D. Postzygotic diploidization of triploids as a source of unusual cases of mosaicism, chimerism and twinning. Hum Reprod 2003 ; $18: 236-42$.

24. Niemann I, Bolund L, Sunde L. Twin pregnancies with diploid hydatidiform mole and co-existing normal fetus may originate from one oocyte. Hum Reprod 2008 ; $23: 2031-5$.

25. Coullin P, Diatta A. L, Boufettal H, et al. The involvement of the transgenerational effect in the high incidence of the hydatidiform mole in Africa. Placenta $2015 ; 36$ : 48-51. 


\section{RÉFÉRENCES}

26. Clagett-Dame M, Knutson D. Vitamin A in reproduction and development. Nutrients $2011 ; 3: 385$ 428.

27. Laanpere M, Altmäe $S$, Stavreus-Evers A, et al. Folate-mediated one-carbon metabolism and its effect on female fertility and pregnancy viability. Nutr Rev $2010 ; 68: 99-113$.

28. Proudhon C, Bourc'his D. Évolution de l'empreinte parentale chez les mammifères. Med Sci (Paris) $2010 ; 26: 497-503$.

29. Kawahara M, Wu Q, Takahashi N, et al. High-frequency generation of viable mice from engineered bi-maternal embryos. Nat Biotechnol $2007 ; 25$ : 1045-50.

30. Parazzini F, La Vecchia C, Mangili G, et al. Dietary factors and risk of trophoblastic disease. Am J Obstet Gynecol 1988 ; $158: 93-9$.
31. Jacobs PA, Hunt PA, Matsuura JS, et al. Complete and partial hydatidiform mole in Hawaii: cytogenetics, morphology and epidemiology. Br J Obstet Gynaecol $1982 ; 89: 258-66$.

32. Matsui $H$, litsuka Y, Yamazawa $K$, et al. Changes in the incidence of molar pregnancies. A population-based study in Chiba prefecture and Japan between 1974 and 2000. Hum Reprod 2003 ; 18 : 172-5.

33. Tillard B. Le placenta : entre oubli familial et investissement médical. Face à Face (revue du laboratoire Société, Santé, Développement) 2004 ; 6 : 48-58.

TIRÉS À PART

J.J. Candelier

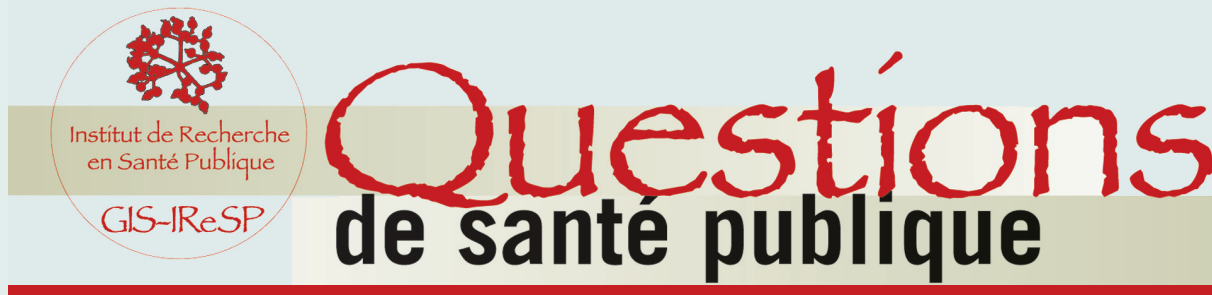

\section{Un nouveau bulletin \\ pour une meilleure \\ visibilité des résultats \\ de la recherche \\ en santé publique}

es résultats de la recherche en santé publique souffrent en France d'un réel manque de visibilité. Ceci concerne aussi bien le monde académique (hors santé publique) que le grand public et les décideurs. Pour pallier ce déficit, l'IReSP a créé un bulletin à large diffusion intitulé "Questions de santé publique ", largement inspiré du bulletin mensuel d'information de l'INED "Populations et sociétés ". L'objectif éditorial est de porter à la connaissance d'un large public (enseignants, étudiants, journalistes, décideurs, milieux de la recherche, asso- ciations, public concerné) les informations les plus récentes concernant des questions importantes de santé publique, rédigées de façon facilement lisible et compréhensible pour des non spécialistes, en garantissant que les informations publiées sont validées scientifiquement. La publication concerne des faits et non des positions. Au-delà de la présentation de résultats, les qualités pédagogiques de Questions de santé publique permettent au lecteur de mieux comprendre comment sont formulées et abordées les questions de santé publique et quelles sont les limites de ces études.

Nom

Prénom

Institution

Fonction

Spécialité

Service

Adresse

Ville

Code postal

Pays

Adresse électronique

à nous retourner par la poste ou par fax au 0149850345

Questions de santé publique

Les Éditions EDK EDP Sciences

109, avenue Aristide Briand

92541 Montrouge Cedex

France

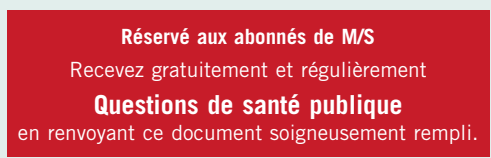

Questions de santé publique est une publication de I'Institut de Recherche en Santé Publique. I Directeur de la publication : Geneviève Chêne. I Rédacteur en chef : Claire-Isabelle Coquin. I Une réalisation des Éditions EDK. 